\title{
SIMPLE IMPELLER SYSTEMS FOR MAINTENANCE OF OIL PALM CULTURE AGGREGATES
}

TARMIZI, A H ${ }^{\star}$; ZAITON, $\mathbf{R}^{\star}$ and ROSLI, $M \mathrm{Y}^{*}$

\begin{abstract}
Scaling up of liquid culture systems generally involves moving from the use of simple shake flasks to bioreactors or specialised vessels; this is costly. A new innovation called the Two-inOne MPOB Simple Impeller (2-in-1 MoSLIM) was developed using commonly available Schott bottles in the laboratory. This system provided simultaneous aeration and agitation (two-inone) in a single device for tissue propagation in liquid culture. The 2-in-1 MoSLIM produced cell aggregates with fresh weight increments of two- to six-fold over 30-40 days. This system was a convenient alternative compared to the conventional shake flask system. Multiplication of cultures in the 2-in-1 MoSLIM did not require any shaker or a big space area. This system with a working volume of $300-700 \mathrm{ml}$ used a simple impeller and a pump for agitation and aeration purposes. However, with the 2-in-1 MoSLIM, media replenishment remained a tedious task. To overcome this, modifications were made to the system to enable media replenishment on-site without the need of a sterile hood. The adaptation of 2-in-1 MoSLIM with an earlier innovation, Fast Transfer Technique (MoFaTT) in Liquid Culture System, resulted in the development of the Simple Impeller with Fast Transfer Technique (SLIM-FaTT) system. This new system can be applied to the liquid culture system of any crop with a potential towards automation.
\end{abstract}

Keywords: oil palm suspension cultures, Two-in-One MPOB Simple Impeller ( 2-in-1 MoSLIM), Simple Impeller with Fast Transfer Technique (SLIM-FaTT) systems, replenishment, proliferation, multiplication.

Date received: 24 August 2015; Sent for revision: 2 September 2015; Received in final form: 19 January 2016; Accepted: 25 February 2016.

\section{INTRODUCTION}

The production of oil palm suspension cultures using the individual shake flask system has been well established (De Touchet et al., 1991; Teixera et al., 1995; Wong et al., 1999; Tarmizi et al., 1999; Tarmizi, 2002). The protocols were developed to produce a reliable supply of regenerable plant tissues. The liquid culture system was also developed to address the inefficiency issues in the micropropagation technique. In liquid medium, the close contact of the

\footnotetext{
* Malaysian Palm Oil Board, 6 Persiaran Institusi, Bandar Baru Bangi, 43000 Kajang, Selangor, Malaysia. E-mail: mizi@mpob.gov.my
}

tissue with the medium stimulates and facilitates the uptake of nutrients and phytohormones, leading to a better growth development. Within the shake flask culture conditions, the growth and multiplication rate of the shoots is enhanced by forced aeration, since continuous shaking of the medium provides sufficient oxygen supply to the tissues, which ultimately leads to their faster growth. According to Gupta and Timmis (2005), somatic embryos development in liquid medium offers tremendous potential as a method for mass propagation as large number of plantlets can be produced inexpensively, easy and quick scale-up can be achieved. They also observed that cultures grown in liquid medium have shown a faster rate 
of growth. Cultures are bathed in liquid, which allows rapid uptake of nutrients by cells and speedy nutrient replacement at the cell surface by diffusion and movement from outlying liquid. Preparation of the liquid medium and handling of shake cultures are simpler than the solid medium (Mehrotera et al., 2007). However, according to Takayama and Akita (2005), the production cost is still high and a large number of culture vessels are required for propagating liquid cultures using the shake flask system. In addition, the conventional method in establishment, maintenance and maturation of liquid cultures is limited due to the size of the flasks or shakers (Tarmizi, 2002). For scaling up the liquid culture system, bioreactors or special commercial flasks were used (Tarmizi et al., 2003) instead of shakers. This system was, however, rather costly, requiring complicated components and with a high maintenance cost.

To address this shortcoming, the Two-in-One MPOB Simple Impeller (2-in-1 MoSLIM) and Simple Impeller with Fast Transfer Technique (SLIMFaTT) were developed as a new process to provide simultaneous aeration and agitation (two-in-one) for the establishment, maintenance and maturation of liquid cultures and fast media transfer.

\section{MATERIALS AND METHODS}

\section{Components of the MPOB 2-in-1 MoSLIM and SLIM-FaTT Systems}

The MPOB 2-in-1 MoSLIM system consisted of the following components: a $500 \mathrm{ml}$ or $1000 \mathrm{ml}$ graduated Schott glass bottle, $1 \mathrm{~m}$ of silicon tubing (Cole-Parmer), vent/ filter devices $64 \mathrm{~mm}(0.22 \mu \mathrm{m}$ : Sartorius), a ratchet clamp (Cole-Parmer), Y-Type connector (Cole-Parmer), a magnetic stirring bar $(8 \mathrm{~mm} \times 50 \mathrm{~mm})$, a screw cap with hole, a top plate (with inlet and outlet openings), a simple air pump and a magnetic stirrer. For the SLIM-FaTT system, additional four graduated Schott glass bottles of $500 \mathrm{ml}$ or $1000 \mathrm{ml}$ capacity with two side arms were required.

\section{Plant Material}

Embryogenic suspensions with aggregate sizes of less than $2 \mathrm{~mm}$ from six clones (derived from elite palms) were used to test the 2-in-1 MoSLIM and SLIM-FaTT systems. The clones used were PL 100 (0.193/50), PL 104 (0.194/455), PL 110 (0.192/2202), PL 127 (0.195/1085), PL 139 (0.366/123) and PL 141 (HDP56/385).

\section{Media}

The liquid MS (Murashige and Skoog, 1962) medium supplemented with $1 \mathrm{mg}$ litre $^{-1}$ nicotinic acid, $0.1 \mathrm{~g}$ litre ${ }^{-1}$ myo-inositol, $0.1 \mathrm{~g}$ litre ${ }^{-1} \mathrm{~L}$-glutamine and $3 \%$ sucrose (castor sugar ) was used (Rohani $e t$ al., 2003). The medium was further supplemented with $1 \mathrm{mg}$ litre $^{-1}$ 2,4-dichlorophenoxyacetic acid (2,4-D) and $0.1 \mathrm{mg}$ litre $^{-1} \alpha$-naphthaleneacetic acid (NAA). This combination of growth regulators was found effective for the oil palm liquid culture system (Tarmizi, 2002). The $\mathrm{pH}$ of the medium was adjusted to 5.7 before autoclaving at $121^{\circ} \mathrm{C}$ for $25 \mathrm{~min}$.

\section{Maintenance of Cultures in the 2-in-1 MoSLIM and SLIM-FaTT Systems}

Approximately $1.60 \mathrm{~g}$ of embryogenic suspension cultures were inoculated into $400 \mathrm{ml}$ of MS medium each, in the 2-in-1 MoSLIM and SLIMFaTT systems. For comparison with the conventional method, suspensions of the same cultures were transferred into individual $100 \mathrm{ml}$ flasks with an inoculum of $0.5 \mathrm{~g}$ suspension per $20 \mathrm{ml}$ of the same medium and agitated on an orbital shaker, and this served as a control for this study. Both systems were incubated in the dark.

\section{Statistical Analysis}

Data was analysed using the analysis of variance (ANOVA) and Duncan New Multiple Range Test (DNMRT) method at $\mathrm{p} \leq 0.05$ for comparison between treatments.

\section{RESULTS AND DISCUSSION}

\section{Development of the 2-in-1 MoSLIM and SLIM-} FaTT for Liquid Culture Micropropagation

2-in-1 MoSLIM. The 2-in-1 MoSLIM consisted of an appropriately sized tubing, a magnetic stirring bar and top plate (Figure 1). The magnetic stirring bar was located inside the lower end of a short tubing and attached to another short tubing (upper part) via a connector. The free end of this short tubing was then attached to the inner port of the top plate. The short tubing with magnetic stirring bar was perforated at appropriate points. This tubing was then placed inside a graduated Schott bottle containing the inoculum and medium. One end of another long tubing was then attached to the outer port of the top plate and connected to a simple pump for aeration. The aeration process which supplies oxygen improves the growth rate and final biomass of cultures (Takayama and Akita, 2005). The Schott bottle was then placed on a magnetic stirrer for agitation (about $80 \mathrm{rpm}$ ). The impeller provided simultaneous agitation and aeration (two-in-one) when the system was switched on. This component was important to facilitate the mixing process which was necessary to ensure equal distribution of cells 


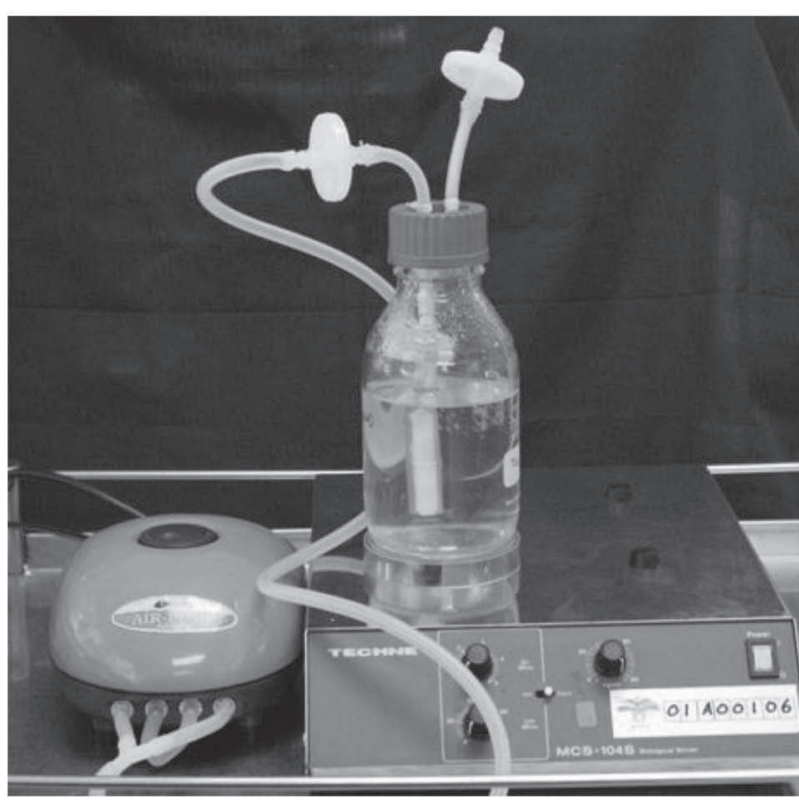

Figure 1. The 2-in-1 MoSLIM for multiplication of culture aggregates.

and / or tissues, and nutrients throughout the liquid phase (Paek et al., 2005).

SLIM-FaTT. Modified Schott bottles with side arms were interconnected with silicon tubings and placed in the culture room. The Schott bottle with cultures was put on the magnetic stirrer for agitation and connected to a simple pump for aeration. The arrangement of the system depends on the usage and nature of the experiment. One possible arrangement is shown in Figure 2. Here, one Schott bottle was designated for culture maintenance (Figure 2i) while two other Schott bottles contained fresh media for maintenance (Figure 2ii) and maturation (Figure 2iii) of cultures. An attached empty Schott bottle (Figure 2iv) served as a reservoir to collect spent culture medium. Clips were attached to the tubings and used as valves to control liquid flow during media replenishment. Filter devices were also attached to each Schott bottle to reduce the pressure build-up within the Schott bottles. Media replenishment was conducted by lifting the Schott bottle containing the appropriate medium at about $15 \mathrm{~cm}$ higher than the base of the receiving vessel at an inclination of about $30^{\circ}$ to $40^{\circ}$ either on a monthly basis or at any desired interval of up to four months. This eliminated the need of having to physically move the vessel to a laminar flow cabinet. We have tested this system with a minimum number of two to four Schott bottles at any one time and the sizes of the Schott bottles ranging from $500 \mathrm{ml}$ to $1000 \mathrm{ml}$. The entire set up was contamination free for at least three months. It is also possible to use larger Schott bottles. Standard configuration of a SLIM-FaTT includes Schott bottles which provide optimum space for the tissue to interact with the medium and to grow under aseptic conditions.

\section{Growth of Cultures in the 2-in-1 MoSLIM and SLIM-FaTT Systems}

2-in-1 MoSLIM. The fresh weight increment of about three- to four-fold was obtained from five oil palm clones multiplied for about 30 days in the 2-in1 MoSLIM system (Figure 3). Some of the plantlets regenerated from this system have been established at the prenursery (Figure 4).

SLIM FaTT. The experimental results revealed that oil palm cultures were able to effectively multiply in the SLIM-FaTT system. The cultures could be maintained for three to four months whilst the replenishment of medium could be done in the culture room at any desired subculture interval, e.g. monthly. A fresh weight increment of about 3- to 16-fold was obtained from three clones tested after about 4 months of maintenance in the system (Figure 5). The fresh weight increment varied amongst clones tested. Comparatively, in this study clone PL

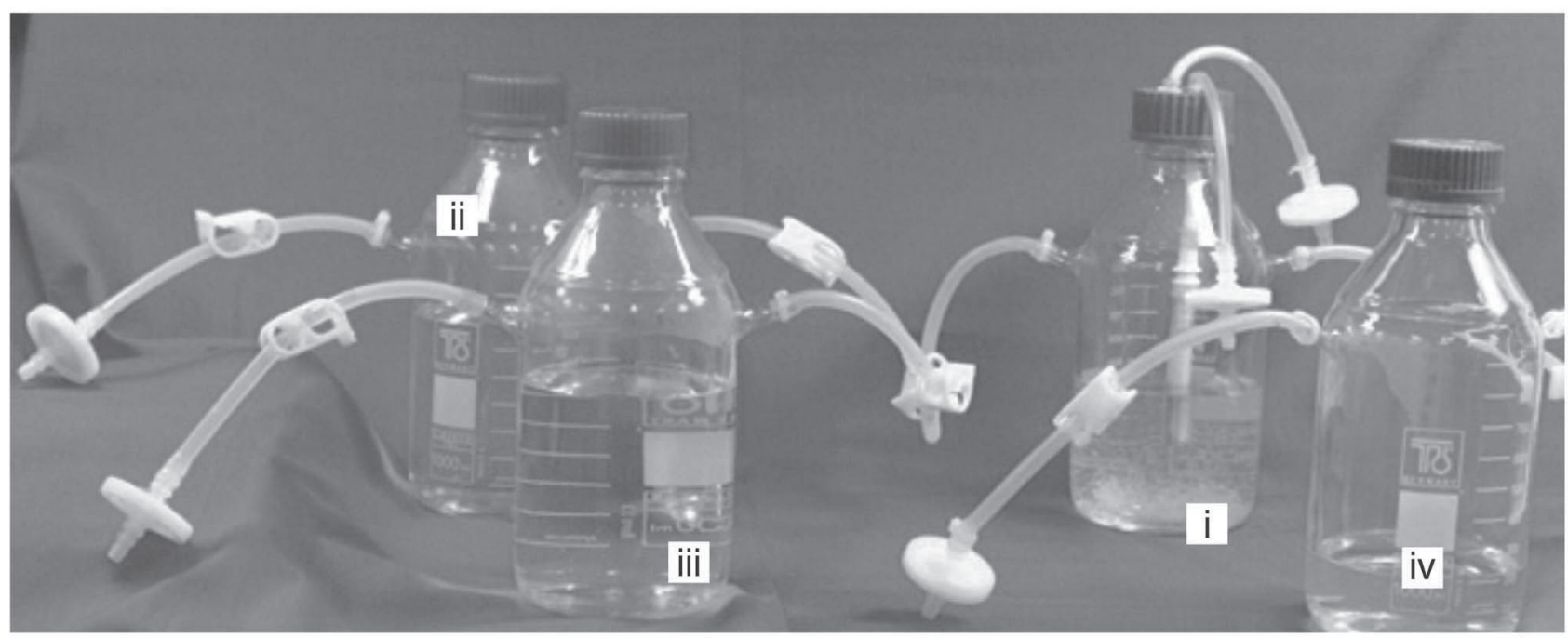

Figure 2. A possible arrangement in SLIM-FaTT. See text for explanation of labelled components. 


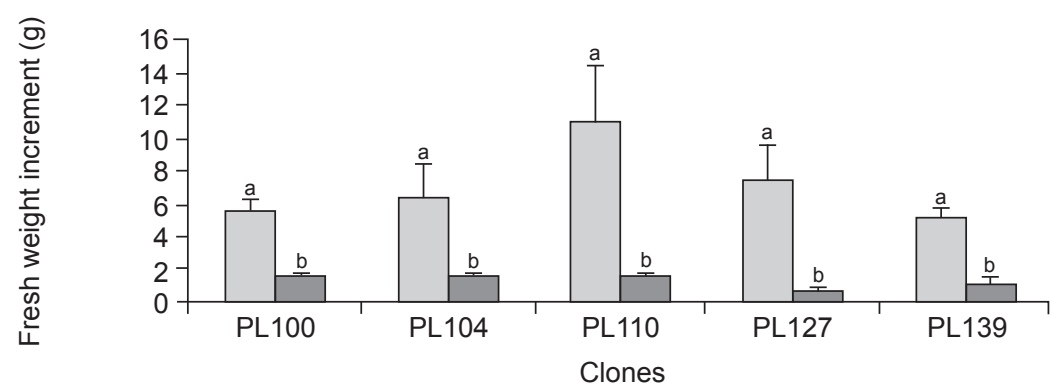

$\square$ MoSLIM $\square$ Shake flasks

Figure 3. Fresh weight increment of cultures from selected clones after about 30 days multiplication in 2-in-1 MoSLIM and shake flask system (conventional method). Bars with the same letters are not significantly different at $P<0.05$ by Duncan New Multiple Range Test (DNMRT).
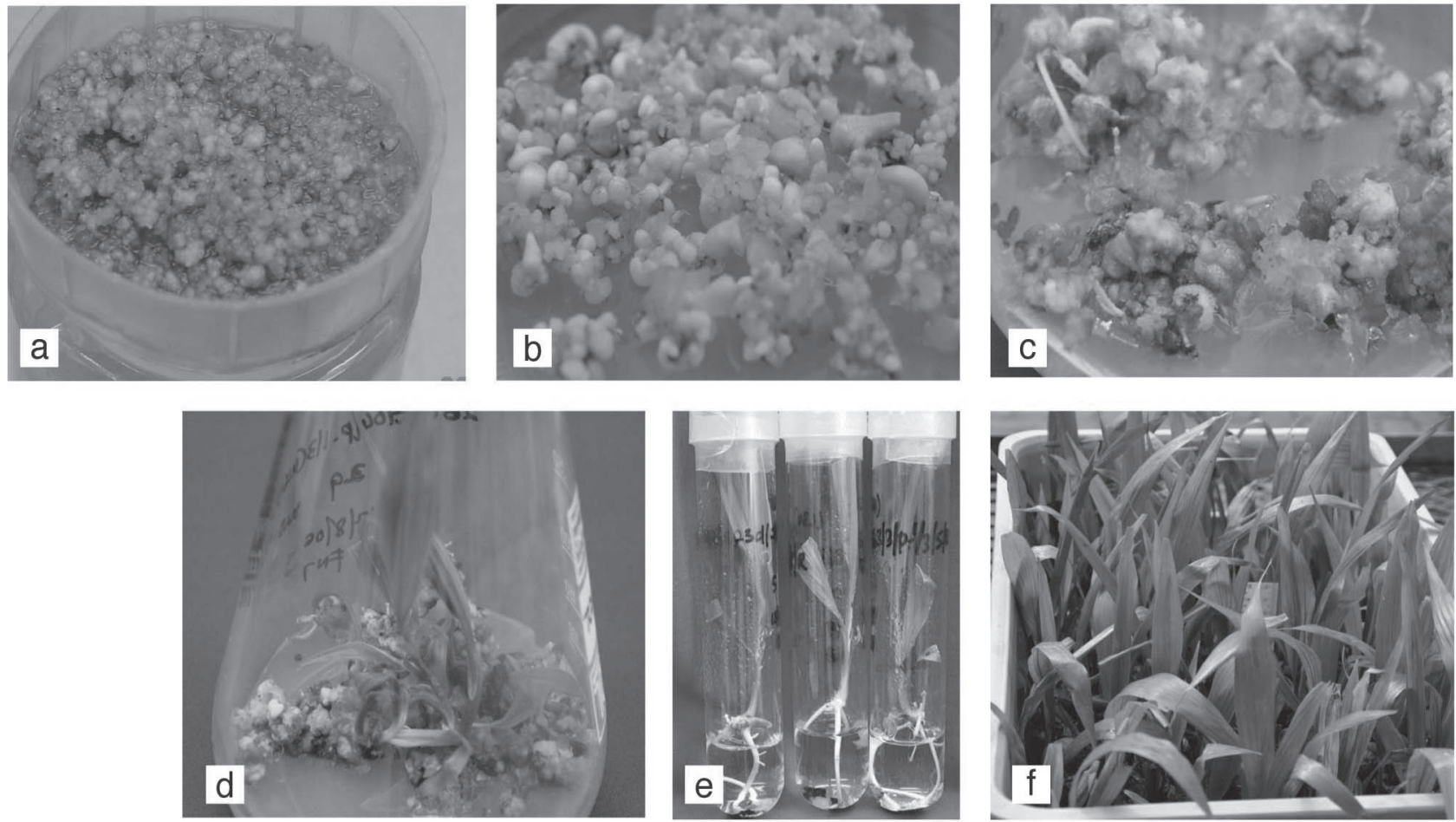

Figure 4. (a) Cell aggregates of clone PL141 generated from 2-in-1 MoSLIM system, (b) regeneration of cell aggregates, (c) development into embryoids, (d) shoot proliferation from polyembryogenic cultures, (e) root development in rooting medium, and ( $f$ ) establishment in nursery.

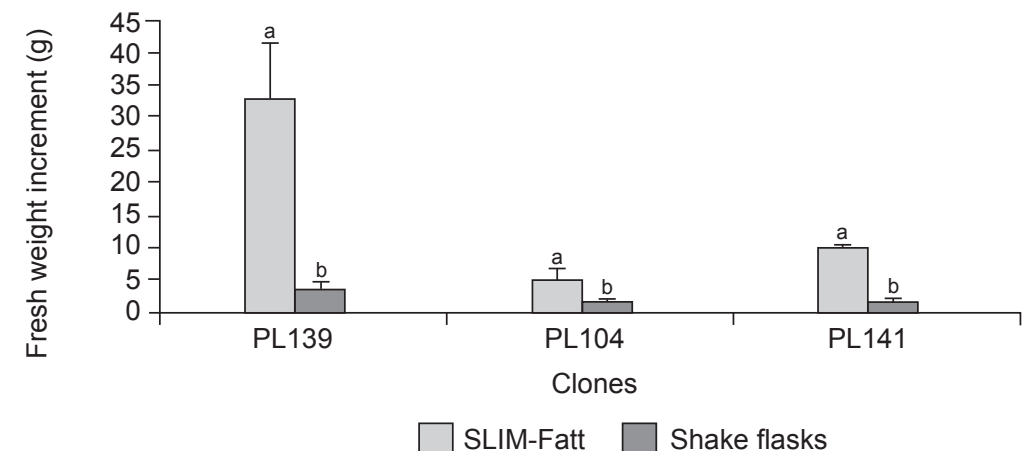

Figure 5. Fresh weight increment of embryogenic cultures from various clones after about four months multiplication in SLIM-FaTT compared with the shake flask system (conventional method). Bars with the same letters are not significantly different at $P<0.05$ by Duncan New Multiple Range Test (DNMRT). 
139 showed the highest fresh weight increment of about 16-fold. Cultures regenerated normally when transferred to solid media and some rooted plantlets have been established at the nursery.

\section{Benefits of the 2-in-1 MoSLIM and SLIM-FaTT Systems}

The usefulness of the 2-in-1 MoSLIM and SLIM-FaTT systems are not only in the higher multiplication/proliferation rate of the cultures but also the convenience of the system compared to the conventional maintenance of cultures in individual shake flasks. The liquid culture system was demonstrated to have significant effects on multiplication rates, morphology of shoots, somatic embryo development, microtubers or bulblets produced in vitro (Preil, 2005). Liquid cultures were also proven to be a good source of starting materials for transformation and protoplast isolation studies (Xiao et al., 2009; Sallets et al., 2015) as well as for secondary metabolite production (Zare et al., 2010; Boonsnongcheep et al., 2010). The inoculum of transformed cells could be multiplied in this system as an alternative to using shake flasks or bioreactors (Sampaio et al., 2010).

Multiplication of cultures in the 2-in-1 MoSLIM and SLIM-FaTT systems did not take up much space and did not require shakers. This system used a simple impeller and a pump for simultaneous agitation and aeration. As it can cater for larger working volumes, e.g. $300 \mathrm{ml}$ to $700 \mathrm{ml}$, more cultures can be produced with this system. Normal multiplication of cultures in $100 \mathrm{ml}$ and $250 \mathrm{ml}$ Erlenmeyer flasks generally only have working volumes of about $20 \mathrm{ml}$ to $100 \mathrm{ml}$, respectively.

Reduction in medium replenishment steps and time saving. As reported earlier, there are at least 10 steps involved in the conventional medium replenishment procedure using the shake flask system during subculturing (Tarmizi and Zaiton, 2005).

However, the 2-in-1 MoSLIM took only eight steps. It began with the opening of the top plate of the Schott bottle with cultures and flame sealing of the mouth of the Schott bottle. Old media was discarded and the Schott bottle resealed. Similar procedure was repeated for the vessel with fresh media. After replenishing the media into the vessel, the lid was returned to its place and a final flame seal of the mouth was done followed by closing of the top plate. Subsequently, the entire process was further reduced to only two steps with the incorporation of SLIM-FaTT. This system was improved merely by either lifting the vessel to discard spent media or for replenishing fresh media through the gravity feed principle to move liquids. The most significant difference between the conventional method of medium replenishment and SLIM-FaTT was the time taken for this process. It took about 11 min to transfer media conventionally using the shake flask system; this is in contrast to 2 min with the 2-in-1 MoSLIM or about a minute for medium replenishment with SLIM-FaTT. This translates to subculturing of more than 30 flasks in an hour using SLIM-FaTT as compared to only five flasks in shake flasks system.

On-site medium replenishment. Using SLIM-FaTT, media replenishment was no longer needed to be carried out under the laminar flow cabinet as the system allowed for on-site manipulations; this was an added advantage. The replenishment of media was done on the magnetic stirrer inside the culture room itself (Figure 6). As there was no movement of cultures from room to the laminar flow cabinet, cultures were not exposed to external environment and the problem of contamination could be reduced, thus minimising the risk of losing important cultures. Contamination of in vitro plant cultures is a critical factor and was discussed in details by Herman (2004) and Smith (2013). Contamination can also rapidly develop and disperse in liquid medium and this is likely to lead to a total loss of the cultures (Preil, 2005).

Practicality of the system. Even though the 2-in-1 MoSLIM and SLIM-FaTT systems were developed for oil palm cultures, it can be applied to any liquid culture system, whether of plant or microbial origins. Since there is no movement of cultures, the media replenishment can be done easily and quickly at any desired time. This will allow proper experimental design to exploit this advantage to the fullest. Quick replenishment at daily, weekly, monthly or even hourly intervals can be done to look at particular effects on culture development. It is not possible or

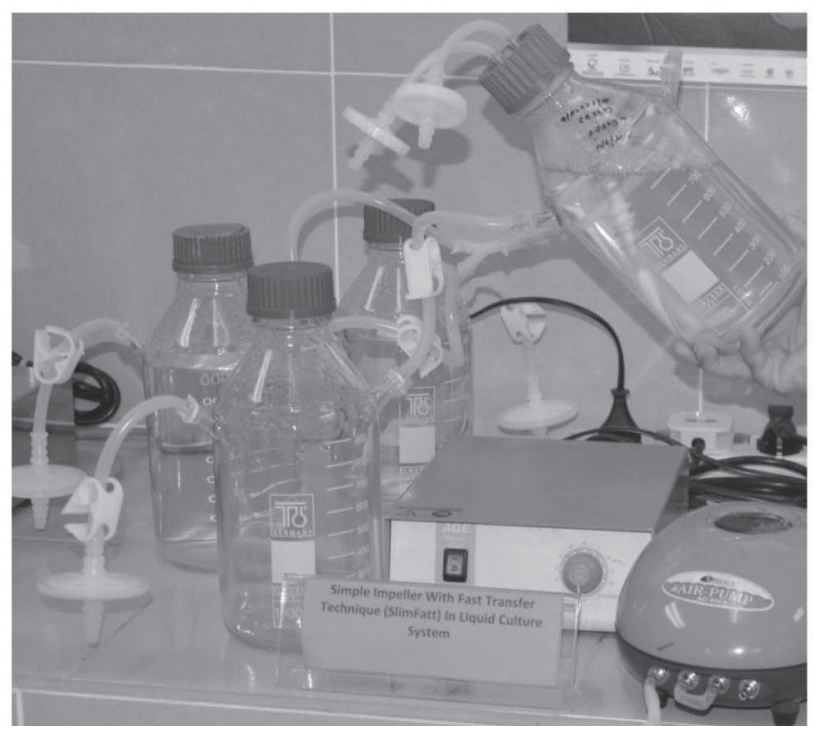

Figure 6. Lifting the Schott bottle to discard spent media or for replenishing fresh media in the culture room. 
practical with the conventional shake flask system. Some cultures require quick media change to avoid browning and the SLIM-FaTT system has simplified this procedure. The use of graduated vessel also enables operators to transfer the required amount of fresh media during replenishment. All these advantages make SLIM-Fatt the practical choice for oil palm or other plant suspension culture systems.

\section{CONCLUSION}

The basic protocol using the shake flask system has been established and the bioreactor technique was also previously developed to further improve the liquid culture system (Tarmizi et al., 2003). MPOB Fast Transfer Technique (MoFaTT) in liquid culture system which was developed earlier (Tarmizi and Zaiton, 2005) was reported to be a convenient method for liquid media replenishment in the shake flask system. However, this technique can only be conducted with the shaker system. The 2-in-1 MoSLIM was developed as an alternative to the shake flask system whereby cultures are maintained on a magnetic stirrer and not on a shaker. Hence, 2-in-1 MoSLIM and SLIM-FaTT, which are both liquid-based, were developed as a rapid and convenient method for liquid media maintenance and maturation of cultures in Schott bottles coupled with efficient media replenishment capability. The system can also be applied to any other liquid culture systems. Various experimental approaches can be designed using the SLIM-FaTT system such as intermittent medium replenishment, on-site application of various exogenous treatments to cultures, etc. Since sugar was reported as one of the limiting growth factors (Paek et al., 2005), an intermittent supply of this component may be introduced into the culture using the SLIMFaTT system. Common problems in liquid culture systems such as browning can be overcome by quick replenishment of fresh media.

Overall, SLIM-FaTT allows a rapid and convenient method to propagate liquid cultures with minimal movement of cultures to and from the laminar flow cabinet as media replenishment can now be done on-site during subculture. An added benefit is that contamination risks are also reduced. This further improves the efficiency of the liquid culture system. Combination of SLIM-FaTT with a simple manifold design for gas delivery at very low flow rate will be the next improvement to the system. Injecting air into suspension at very low flow rate has proven effective in plant tissue culture propagation (Shaw et al., 2012). Besides simple impeller system, further modification by using an airlift or simple tubing system for aeration could also be incorporated in both 2-in-1 MoSLIM and SLIM-FaTT systems. The application of airlift bioreactor system was proven effective in the multiplication of American chestnut cultures (Kong et al., 2014). Furthermore, this technology can be further exploited by semi or fully-automating the oil palm clonal production process.

\section{ACKNOWLEDGEMENT}

The authors wish to thank the Director-General of MPOB for permission to publish the article. Thanks are also due to Dr Ooi Siew Eng and Zamzuri Ishak for their helpful comments on the manuscript. Support and encouragement from Dr Ravigadevi Sambanthamurthi, the former Director of Advanced Biotechnology and Breeding Centre, MPOB; the Head of Breeding and Tissue Culture Unit, MPOB and technical assistance from the Tissue Culture staff are also gratefully acknowledged.

\section{REFERENCES}

BOONSNONGCHEEP, P; KORSANGRUANG, S; SOONTHORNCHAREONNON, N; CHINTAPAKORN, Y; SARALAMP, P and PRATHANTURARUG, S (2010). Growth and isoflavonoid accumulation of Pueraria candollei var. candollei and $P$. candollei var. mirifica cell suspension cultures. Plant Cell, Tissue and Organ Culture, 101: 119-126.

DE TOUCHET, B; DUVAL, $\mathrm{Y}$ and PANNETIER, C (1991). Plant regeneration from embryogenic suspension cultures of oil palm (Elaeis guineensis Jacq.). Plant Cell Report, 10: 529-532.

GUPTA, P K and TIMMIS, R (2005). Mass propagation of conifer trees in liquid cultures progress towards commercialization. Plant Cell, Tissue and Organ Culture, 81: 339-346.

HERMAN, E B (2004). Microbial 'contaminants' in plant tissue cultures: solutions and opportunities 1996-2003. Recent Advances in Plant Tissue Culture VIII. Agritech Consultants, Inc., Shrub Oak. p. 115.

KONG, L; HOLTZ, C T, NAIRN, C J and HOUKE, H (2014). Application of airlift bioreactors to accelerate genetic transformation in American chestnut. Plant Cell, Tissue and Organ Culture, 117: 39-50.

MEHROTRA, S; GOEL, M K; KUKREJ, A and MISHRA, B N (2007). Efficiency of liquid culture systems over conventional micropropagation: a progress towards commercialization. African J. Biotechnology, Vol. 6: 1484-1492.

MURASHIGE, T and SKOOG, F (1962). A revised medium for rapid growth and bioassays with 
tobacco tissue cultures. Physiologia Plantarum,15: 473-497.

PAEK, K Y; CHAKRABARTY, D and HAHN, E J (2005). Application of bioreactor systems for large scale production of horticultural and medicinal plants. Liquid Culture System for in vitro Plant Propagation 2005 (Hvoslef-Eide, A K and Preil, W eds.) Springer, The Netherlands. p. 95-117.

PREIL, W (2005). General introduction: a personal reflection on the use of liquid media for in vitro culture. Liquid Culture System for in vitro Plant Propagation 2005 (Hvoslef-Eide, A K and Preil, W eds.). Springer, The Netherlands. p. 95-117.

ROHANI, O; ZAMZURI, I and TARMIZI, A H (2003). Oil palm cloning: MPOB protocol. $M P O B$ Technology No. 26.

SALLETS, A; DELIMOY, D and BOUTRY, M (2015) Stable and transient transformation of Artemisia annua suspension cells. Plant Cell, Tissue and Organ Culture, 120: 157-164.

SAMPAIO, P N; NETO, H; POEJO, P; SERRAZINA S M and PAIS, S M (2010). Overexpression and characterization of cyprosin B in transformed suspension cells of Cynara cardunculus. Plant Cell Tissue and Organ Culture, 101: 311-321.

SHAW, S; CURTIS, M; FLOREZ, S; LARSEN, J and CURTIS, W R (2012). An improved temporary immersion bioreactor design for plant tissue culture propagation. In vitro Cellular $\mathcal{E}$ Developmental Biology Vol. 48: S39.

SMITH, R H (2013). Contamination. Plant Tissue Culture and Techniques and Experiments. Academic Press. p. 53-63.

TAKAYAMA, S and AKITA, M (2005). Practical Aspects of bioreactor application in mass propagation of plants. Liquid Culture System for in vitro Plant Propagation 2005 (Hvoslef-Eide, A K and Preil, W eds.). Springer, The Netherlands. p. 61-79.
TARMIZI, A H; NORAZLINA, N; ZAITON, R and CHEAH, S C (1999). Establishment of oil palm embryogenic suspension cultures from calli derived from various sources. Proc. of the 11 National Biotechnology Seminar. Jointly organised by MARDI and NBD, Melaka. p. 381-382

TARMIZI, A H (2002). Oil palm liquid cultureMPOB protocol. MPOB Information Series No. 138.

TARMIZI, A H; NORJIHAN, M A; SAMSUL KAMAL, R; ZAITON, R and CHEAH, S C (2003). Mass propagation of oil palm planting materials using liquid culture and bioreactor technology. Proc. of the PIPOC 2003 International Palm Oil Congress Agriculture Conference. MPOB, Bangi. p. 130-144.

TARMIZI, A H and ZAITON, R (2005). MPOB Fast Transfer Technique (MoFaTT) in oil palm liquid culture system. MPOB Information Series No. 261.

TEIXERA, J B; SONDAHL, M R; NAKAMURA, T and KIRBY, E G (1995). Establishment of oil palm suspensions and plant regeneration. Plant Cell, Tissue and Organ Culture, 40: 105-111.

WONG, G; CHONG, S P; TAN, C C and SOH, A C (1999). Liquid suspension culture - a potential technique for mass production of oil palm clones. Proc. of the 1999 PORIM International Palm Oil Congress. PORIM, Bangi. p. 3-11.

XIAO, W; HUANG, X; GONG, Q; DAI, XM; ZHAO, J T; WEI, Y R and HUANG, X L (2009). Somatic hybrids obtained by asymmetric protoplast fusion between Musa Silk cv. Guoshanxiang (AAB) and Musa acuminate cv. Mas (AA). Plant Cell, Tissue and Organ Culture, 97: 313 - 321.

ZARE, K; NAZEMIYEH, H; MOVAFEGHI, A; KHOSROWSHAHLI, M; MOTALLEBI-AZAR, A; DADPOUR, M and OMIDI, Y (2010). Bioprocess engineering of Echium italicum L.: induction of shikonin and alkannin derivatives by two-liquidphase suspension cultures. Plant Cell, Tissue and Organ Culture, 100: 157-164. 